\title{
Tunnelling and instantons
}

\author{
Jérémy Le Deunff ${ }^{1}$, Amaury Mouchet ${ }^{1}$ \\ 'Laboratoire de Mathématiques et de Physique Théorique, Université François Rabelais - CNRS (UMR 6083), \\ Fédération Denis Poisson, Parc de Grandmont 37200 Tours, France.
}




\title{
Tunnelling and instantons
}

\author{
Jérémy Le Deunff ${ }^{1}$, Amaury Mouchet ${ }^{1}$ \\ 'Laboratoire de Mathématiques et de Physique Théorique, Université François Rabelais - CNRS (UMR 6083), \\ Fédération Denis Poisson, Parc de Grandmont 37200 Tours, France.
}

\section{Introduction:}

Tunnelling can be characterised by the splitting between nearly degenerate doublets of energy. Semiclassically, it can be computed using Herring's formula or for simple situations with the help of instanton techniques. The present work proposes an extension of the later for more general situations involving only traces of the evolution operator that are analytically continued in the complex time domain. We will apply it to three examples, in particular for the resonant case for which the instanton approach, where the time is forced to be purely imaginary, is not sufficient.

\section{Tunnelling splittings:}

Using a Hamiltonian with a two fold symmetry, it is possible to construct a good approximation of the tunnelling splitting with the help of complex time $T=|T| \mathrm{e}^{-}$

$$
\Delta E_{0} \simeq \Delta_{0}(T)=\frac{2 \hbar}{i T} \frac{\operatorname{Tr}(\hat{S} \widehat{U}(T))}{\operatorname{Tr}(\widehat{U}(T))}
$$

for the ground state for large enough $\operatorname{Im}(T)(\theta \simeq \pi / 2)$.

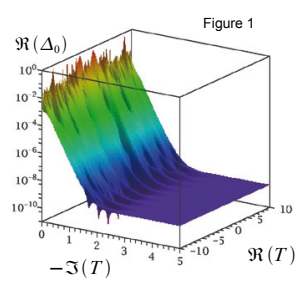

This formula can be generalised to an arbitrary doublet (small $\theta$ ):

$$
\Delta E_{n} \simeq\left|\Delta_{n}(T)\right|=\left|\frac{2 \hbar}{i T} \frac{\operatorname{Tr}\left(\widehat{\Pi}_{n} \hat{S} \widehat{U}(T)\right)}{\operatorname{Tr}\left(\widehat{\Pi}_{n} \widehat{U}(T)\right)}\right|
$$

$\widehat{\Pi}_{n}$ mimics the projector of the doublet state.

Through path integral expressions of the traces, such formulae provide a natural semiclassical expansion in terms of complex (half) periodic orbits.

The strategy consists in retaining dominant complex orbits that connect the two symmetric tori with a complex time path chosen in order to to keep one real canonical variable.

It leads to a formula, applicable to any general symmetric Hamiltonian. Here, the sum is made over all dominant orbits $o$ at energy $E_{n}$

$$
\left|\Delta_{n}(T)\right|_{\hbar \rightarrow 0}^{\sim}\left|\frac{\hbar}{T} \sum_{o} A_{o}\left(E_{n}, T\right) \mathrm{e}^{i \Re\left(S_{o}\right) / \hbar-\Im\left(S_{o}\right) / \hbar}\right|
$$

\section{The double well:}

Complex Hamilton's equations indicate a natura descending staircase shape for the time path (fig. 3a).

The position $q$ is kept real while $p$ is either real or imaginary (inversion of the potential). (fig.2a,b)

It is natural to represent the dominant classical orbits which are a concatenation of three trajectories in each region in a 3-D phase space (q, $\operatorname{Re}(p), \operatorname{Im}(p))$. (fig. $2 c, 3 b)$

The semiclassical expansion leads to the Landau-Lifschitz formula:

$$
\Delta_{n \rightarrow 0} \frac{\hbar \omega}{\pi} \mathrm{e}^{-\tilde{S}_{\varepsilon}\left(E_{n}\right) /(2 \hbar)}
$$

\section{The pendulum: $V(q)=-\gamma \cos (q)$}

This is an example of (dynamical) tunnelling between a classical rotation and its inverse.

The same shape of the time path than the double well is kept but the real variable is now the impulsion p. (fig. 3a)

The natural representation is thus the 3-D phase space $(p, \operatorname{Re}(q), \operatorname{Im}(q))$. (fig. 4a,b)

An imaginary position does not involve a potential inversion but another transformation:

The method permits to find an explicit expression of the tunnelling splitting for large $n$ :

$$
\Delta_{n \sim \sim 00} \frac{1}{\pi n^{4 n-1}}\left(\frac{\mathrm{e}}{2}\right)^{4 n} \hbar^{2}\left(\frac{\gamma}{\hbar^{2}}\right)^{2 n}
$$

Figure 5 shows the "exact" splitting and the semiclassical approximation (fig. 5a). The validity of the prefactor is controlled on (fig. 5b) and we can see that it tends to the "exact" one in the semiclassical limit.
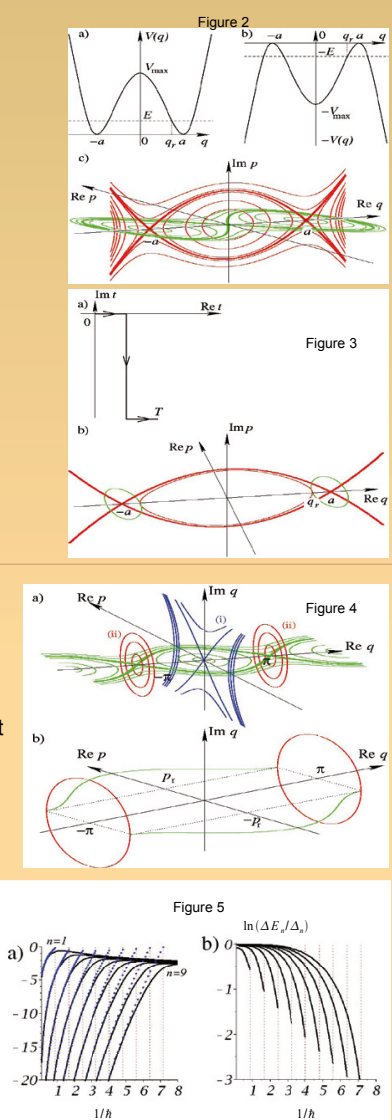

\section{Resonant tunnelling and Fabry-Pérot effect:}

When there is a deeper central well, resonant tunnelling occurs.

To keep the position q real, we need a two step time path (fig. $6 \mathrm{~d}$ ) and orbits are represented as a concatenation of five trajectories connected in the 3-D phase space (q, $\operatorname{Re}(p), \operatorname{Im}(p))$. (fig. $6 c, e)$

The semiclassical expression is compared to the "exact" splitting on (fig. 7) and appears as a very good approximation

$$
\begin{aligned}
& \left|\Delta_{n}(T)\right|_{\hbar \rightarrow 0}^{\sim}\left|\frac{2 \hbar}{T} \mathrm{e}^{-\tilde{S}_{c}\left(E_{n}\right) / \hbar} F_{n}(\hbar, T)\right| \\
& \text { with } \\
& \left|F_{n}(\hbar, T)\right| \underline{\underline{\text { def }}}\left|=\sum_{\substack{\left(\omega_{n}, \omega_{m}\right) \text { pos. int. such that } \\
\omega_{r} T_{r}\left(E_{n}\right)+\omega_{m} T_{m}\left(E_{n}\right)=R(T)}}\left(\omega_{r}+1\right) \mathrm{e}^{i \omega_{r}\left[\tilde{S}_{r}\left(E_{n}\right) / \hbar-\pi\right]+i \omega_{m}\left[\tilde{S}_{m}\left(E_{n}\right) / \hbar-\pi\right]}\right| \\
& \sim \frac{1}{\left|\sin \left(\left(\tilde{S}_{r}\left(E_{n}\right) / \hbar-\pi\right)-\left(\tilde{S}_{m}\left(E_{n}\right) / \hbar-\pi\right)\right)\right|}
\end{aligned}
$$

The resonance appears when the energy of a state mainly localised in the central well becomes nearly degenerate with the energy doublet in the lateral wells.

\section{Conclusion:}

This strategy gives an intuitive interpretation of the splitting in terms of complex orbits and is more efficient than the standard instanton method, because it takes full advantage of working with an arbitrary complex time path (not only a purely imaginary one), in particular for the resonant tunnelling where the real part of the time cannot vanish. Since it does not rely on the analytic continuation of the quasimodes deeply in the forbidden region, it may provide a deeper understanding of tunnelling in chaotic cases. 